\title{
Ways to Measuring Quality of Life in Mental Health
}

Mirna Fontelene de Oliveira1, Régia Christina Moura Castro ${ }^{2}$, Cinthia Gondim Pereira Calou ${ }^{2}$, Mara Fontenele de Oliveira2, Priscila de Souza Aquino², Cláudio Gleidiston Lima da Silva',

\section{Abstract}

As quality is of life considered as a measure of clinical outcome that prioritizes client assessment itself and the effects of a disease, a life change or a treatment has on their daily life and their level of satisfaction and well-being, their evaluation allows obtain a safe parameter for implementing clinical interventions that may have more positive impact on the lives of these people. It is believed that investigate the QoL of a given population is a strategy that will enable to broaden the understanding of the problems experienced by patients in order to facilitate effective future interventions, improving the quality of lived days. This information can also be used to identify patients at higher risk of problems and thus anticipate interventions, contributing to health promotion thereof. Nevertheless, there is currently a growing interest in transforming the QoL in a quantitative measure. To this end, the measurement of quality of life through the perception of the patient has been recommended. Some studies indicate that the measurement of quality of life the mental patient is an indicator of the care he receives and that this issue should be included in the assessment and care planning. There are few studies witch accessed the influence of health intervention on patients 'quality of life, anxiety, and depressive symptom levels. With this brief contribution, we hope can deepen discussion regards public health and mental wellbeing, as well as the options of measurement instruments to assess mental health interventions and thus able to gather more arguments to answer the following question: What's the best way for measuring quality of life in Mental health?

Over the last decade, the Department of Mental Health and Substance Abuse at the World Health Organization (WHO) has incrementally built a helpful model for conceptualizing public mental health in a global context, which has most recently been incorporated into the WHO's Mental Health Action Plan 2013-2020 [1, 2].

Camilla Barros Meireles ${ }^{1}$, Laís Chaves Maia1, Victória Aline Linhares Miná1, Bernardo Nogueira Faé', Matheus Felipe Aguiar Santos ${ }^{1}$, Maria do Socorro Vieira dos Santos ${ }^{1}$, Janaina Batista Pereira ${ }^{3}$, Hellen Cruz Caldas Lins ${ }^{3}$, Modesto Leite Rolim Neto', Ana Karina Bezerra Pinheiro ${ }^{2}$

1 Department of Medicine, Federal University of Cariri, Barbalha, Ceará, Brazil..

2 Department of Nursing, Federal University of Ceará, Fortaleza, Brazil.

3 Post-graduation Program in Health Sciences, Faculty of Medicine of $A B C$

Contact information:

Modesto Leite Rolim Neto.

”- modestorolim@yahoo.com.br

\section{Keywords}

Quality of life, Mental health,

Treatment 
The WHO has defined health as 'a state of complete physical, mental and social well-being and not merely the absence of disease or infirmity' [3]. In 2001, the WHO described mental health as 'a state of well-being in which the individual realizes his or her own abilities, can cope with the normal stresses of life, can work productively and fruitfully, and is able to make a contribution to his or her community' [4].

Although this is a helpful start, further evidence and conceptual clarity were clearly needed. In response, the WHO published two key reports about the prevention of mental disorders (5) and promoting mental health (6), in which they recognized three ideas central to the improvement of health:

$\checkmark$ Mental health is an integral part of health.

$\checkmark$ Mental health is more than the absence of illness.

$\checkmark$ Mental health is intimately connected with physical health and behavior.

Additionally, in a recent publication about public mental health, has described the importance of both treating mental health as equal to physical health and of focusing on the needs and safety of people with mental illness [7]. In front of this, one way for to achieve this goal is to research the Quality of Life (QoL) of this patients.

We highlight that the prolongation of life expectancy boosted health professionals to think in ways of measuring how people live those extra years and later on measures of quality of life [8].

As quality is of life considered as a measure of clinical outcome that prioritizes client assessment itself and the effects of a disease, a life change or a treatment has on their daily life and their level of satisfaction and well-being, their evaluation allows obtain a safe parameter for implementing clinical interventions that may have more positive impact on the lives of these people [8].

It is believed that investigate the QoL of a given population is a strategy that will enable to broaden the understanding of the problems experienced by patients in order to facilitate effective future interventions, improving the quality of lived days. This information can also be used to identify patients at higher risk of problems and thus anticipate interventions, contributing to health promotion thereof [9].

Nevertheless, there is currently a growing interest in transforming the QoL in a quantitative measure. To this end, the measurement of quality of life through the perception of the patient has been recommended [10].

Some studies indicate that the measurement of quality of life the mental patient is an indicator of the care he receives and that this issue should be included in the assessment and care planning [11]. There are few studies witch accessed the influence of health intervention on patients 'quality of life, anxiety, and depressive symptom levels [12].

From a reduced number of scientific evidence published in the international literature that addresses the measurement of quality of life before and after interventions for mental health, we would like to highlight the following instruments:

$\checkmark$ Short version the World Health Organization Quality of Life Assessment (WHOQOL-BREF) [13];

$\checkmark$ Brief Symptom Inventory (Subscales somatization, anxiety and depression) [14];

$\checkmark$ The Satisfaction with Life Scale (SWLS) [15];

$\checkmark$ Scale of Self-Esteem (Rosenberg Scale) [16] ;

$\checkmark$ The State-Trait Anxiety Inventory (STAI) [17];

$\checkmark$ The Center for Epidemiologic Studies Depression Scale (CES-D) [18];

$\checkmark$ The 24-item Hamilton Depression Rating Scale [19];

$\checkmark$ The Positive and Negative Syndrome Scale (PANSS) [20];

$\checkmark$ Pittsburgh Sleep Quality Index [21];

In a recent organizational documentation was published the Public Mental Health Priorities: mental illness prevention; mental health promotion; and treatment, recovery and rehabilitation [7]. Re- 
garding the prevention of mental illness, interventions like music therapy can be use for improve quality of life and prevent of psychological and somatic symptoms in patients with controlled mental health, as described in a Korean study about hwabyung, a syndrome related to Korean culture [22]. To evaluate the effectiveness of this intervention the following instruments were used: The StateTrait Anxiety Inventory (STAI), The Center for Epidemiologic Studies Depression Scale (CES-D) and the WHOQOL-BREF [22].

From the perspective of Mental Health Promotion, some studies published recently targeted to a sample of elderly, in one this was applied a group psychotherapy and investigated the effectiveness of this group on four domains of quality of life [23], in another was examined the effects of yoga intervention on quality of life and sleep quality of this population [24]. To check the effectiveness of interventions, the first study used the following instruments: The 24-item Hamilton Depression Rating Scale and the WHOQOL-BREF [23]. The second study used: Pittsburgh Sleep Quality Index and WHOQOL-BREF [24].

With respect to Treatment, and Recovery Rehabilitation of severe mental illness, we can cite a quasi-experimental study that evaluates the impact of psycho-educational intervention of Quality of Life in patients suffering from schizophrenia. To check the validity of the intervention, the following measurement instruments were used: Hamilton Rating Scale for Depression and WHOQOL-BREF [25].

The majority of cited researchers argue that questionnaires used have good psychometric properties and are recommended to evaluate the effectiveness of mental health interventions [22, 23, 24, 25].

With this brief contribution, we hope can deepen discussion regards public health and mental wellbeing, as well as the options of measurement instruments to assess mental health interventions and thus able to gather more arguments to answer the following question: What's the best way for measuring quality of life in Mental health?

\section{Conflict of interest}

The authors declare no conflict of interest.

\section{Role of the funding source}

None.

\section{Authors Contributions}

Seven independent researchers, $(\mathrm{MO}, \mathrm{RC}, \mathrm{CC}, \mathrm{MO}$, PA, CS, and CM) conducted a literature research. Any discrepancies between the seven reviewers who, blind to each other, examined the studies for the possible inclusion were resolved by consultations with three senior authors (MR, SS, AP). Other six researchers (LM, VM, MS, SS, JC, and BF) correlated these data information and wrote the Short Communication.

\section{Acknowledgements}

We were supported by: Scientific Writing Lab, Medicine School -Federal University of Cariri- UFCA Research Group: Suicidology-Federal University of Ceará -UFC/National Council for Scientific and Technological Development-CNPq- a body linked to the Ministry of Science, Technology and Innovation to encourage research in Brazil.

\section{References}

1. World Health Organization. Risks to Mental Health: An Overview of Vulnerabilities and Risk Factors. Geneva: WHO; 2012.

2. World Health Organization. WHO Mental Health Action Plan 2013-2020. Geneva: World Health Organization 2013.

3. World Health Organization. Basic Documents. 43rd Edition. Geneva: WHO; 2001.

4. World Health Organization. Strengthening Mental Health Promotion (Fact Sheet No. 220). Geneva: WHO; 2001.

5. World Health Organization. Prevention of Mental Disorders: Effective interventions and policy options. Summary Report. A Report of the World Health Organization, Department of Mental Health and Substance Abuse in collaboration with The Prevention Research Centre of the Universities of Nijmegen and Maastricht. Geneva: World Health Organization 2004. 
6. World Health Organization. Promoting Mental Health: Summary Report. A Report of the World Health Organization, Department of Mental Health and Substance Abuse in collaboration with the Victorian Health Promotion Foundation and the University of Melbourne. Geneva: World Health Organization 2005.

7. Davies, S., \& Mehta, N. Public mental health: evidence based priorities. Treatment, recovery and rehabilitation. Annual Report of the Chief Medical Officer, Public Mental Health Priorities: Investing in the Evidence (UK): 2013; 320p.

8. Fleck MPDA. The World Health Organization instrument to evaluate quality of life (WHOQOL-100): characteristics and perspectives. Ciência \& Saúde Coletiva 2000; 5(1): 33-38.

9. Hacker ED, Ferrans CE. Quality of life immediately after peripheral blood stem cell transplantation. Cancer nursing 2003; 26(4): 312-22.

10. Hill PD, Aldag JC, Hekel B, Riner G, Bloomfield P. Maternal postpartum quality of life questionnaire. Journal of Nursing Measurement 2006; 14(3): 205-20.

11. Connell J, O'Cathain A, Brazier J. Measuring Quality of Life in Mental Health: Are we asking the right questions?. Social Science \& Medicine. 2014

12. Donker JM, de Vries J, de Lepper CC, Wielders D, Ho GH, Mulder PG, van der Laan L. A Novel Finding: The Effect of Nurse Practitioners on the Relation to Quality of Life, Anxiety, and Depressive Symptoms in Vascular Surgery. Annals of vascular surgery 2014; 28(3): 644-50.

13. Harper H, Power M, Group TW. Development of the World Health Organization WHOQOL-BREF quality of life assessment. Psychol Med 1998; 28: 551-58.

14. Derogatis LR, Melisaratos N. The brief symptom inventory: an introductory report. Psychological medicine 1983; 13(03): 595605.

15. Diener ED, Emmons RA, Larsen RJ, Griffin S. The satisfaction with life scale. Journal of personality assessment 1985; 49(1): 71-75.

16. Robins RW, Hendin HM, Trzesniewski KH. Measuring global selfesteem: Construct validation of a single-item measure and the Rosenberg Self-Esteem Scale. Personality and social psychology bulletin 2001; 27(2): 151-61.

17. Marteau TM, Bekker H. The development of a six囚item short》 form of the state scale of the Spielberger State-Trait Anxiety Inventory (STAI). British Journal of Clinical Psychology 1992; 31(3): 301-06

18. Radloff L. The CES-D Scale: A self-report depression scale for research in the general population. Appl Psychol Meas 1997; 1 : $368-401$

19. Hamilton MA. A rating scale for depression. J Neurol Neurosurg Psychiatry 1960; 23: 56 -62

20. Kay SR, Flszbein A, Opfer LA. The positive and negative syndrome scale (PANSS) for schizophrenia. Schizophrenia bulletin 1987; 13(2): 261
21. Pallesen N, Omvik S, Matthiesen B. Pittsburgh sleep quality index. TIDSSKRIFT-NORSK PSYKOLOGFORENING 2005; 42(8): 714.

22. Park JS, Park $\mathrm{S}$, Cheon $\mathrm{CH}$, Jang $\mathrm{BH}$, Lee $\mathrm{SH}$, Lee $\mathrm{SH}, \ldots$ Ko SG. Effect of oriental medicine music therapy on patients with Hwabyung: a study protocol for a randomized controlled trial. Trials 2012; 13(1): 161

23. Wang CC, Tzeng DS, Chung WC. The effect of early group psychotherapy on depressive symptoms and quality of life among residents of an apartment building for seniors. Psychogeriatrics 2014; 14(1): 38-46.

24. Hariprasad VR, Sivakumar PT, Koparde V, Varambally S, Thirthalli J, Basavaraddi MVI, Gangadhar BN. Effects of yoga intervention on sleep and quality-of-life in elderly: A randomized controlled trial. Indian journal of psychiatry 2013; 55(Suppl 3): S364.

25. Silva RCRD, Saute JAM, Silva ACFD, Coutinho ACDO, SaraivaPereira ML, Jardim L B. Occupational therapy in spinocerebellar ataxia type 3: an open-label trial. Brazilian Journal of Medical and Biological Research 2010; 43(6): 537-42.

\section{Comment on this article:}

\section{A $B$ in $8+\mathbf{S} P$}

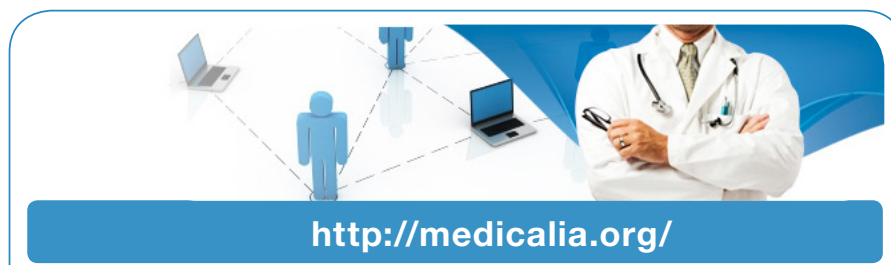

Where Doctors exchange clinical experiences, review their cases and share clinical knowledge. You can also access lots of medical publications for free. Join Now!

\section{Publish with iMedPub}

\section{http://www.imed.pub}

International Archives of Medicine is an open access journal publishing articles encompassing all aspects of medical science and clinical practice. IAM is considered a megajournal with independent sections on all areas of medicine. IAM is a really international journal with authors and board members from all around the world. The journal is widely indexed and classified Q1 in category Medicine. 